correctional work design, including around-the-clock operations and routine pressure to participate in overtime work may have additional consequences for health and retention of an ageing correctional workforce. In this study, we examined the implications of shift work and extended work hours for officers' ability to maintain workability, health, and well-being in the face of stressful work demands, with attention focused on officer age (chronological age and psychological age) as a risk variable for increased negative outcomes.

Methods As part of a Total Worker Health intervention study, corrections officers at a state correctional system in the northeastern USA participated in Wave $1(n=335)$ or Wave 2 $(n=260)$ of a survey that assessed physical and psychosocial work demands, work schedules, and several aspects of personal health and well-being. ANCOVA and moderated multiple regression analyses were used to examine the interactive effects of work schedule and age on relationships between work demands and worker health and well-being. An additional data collection in the same correctional system has recently been completed and will form the basis for follow-up analyses.

Results Initial findings indicate that corrections officers in this sample are at risk of several negative physical and mental health outcomes (e.g., obesity, depressive symptoms, burnout). Psychosocial features of corrections work, including work schedules, interacted with correctional officer age, with stronger negative consequences for several aspects of personal health and well-being among chronologically and psychologically older officers.

Conclusion In the face of an ageing corrections workforce, our findings suggest that particular attention should be paid to interventions that mitigate the impact of nightwork and overtime on the health and well-being of older officers.

\section{C CIRCADIAN AND SLEEP HOMEOSTATIC INTERVENTION STRATEGIES FOR OLDER SHIFT WORKERS}

\begin{abstract}
${ }^{1,2}$ Jeanne F Duffy, ${ }^{1,2}$ Wei Wang, ${ }^{1}$ Audra S Murphy, ${ }^{1,2}$ Cheryl M Isherwood, ${ }^{1,2,3}$ Evan D Chinoy, ${ }^{1,2,4}$ Jee Hyun Kim, ${ }^{1,2,5}$ Min Ju Kim. ${ }^{1}$ Division of Sleep and Circadian Disorders, Brigham and Women's Hospital, Boston, MA, USA; ${ }^{2}$ Division of Sleep Medicine, Harvard Medical School, Boston, MA, USA; ${ }^{3}$ Warfighter Performance Department, Naval Health Research Centre, San Diego, CA, USA; ${ }^{4}$ Dept. of Neurology, Dankook University College of Medicine and Hospital, Cheonan, Republic of Korea; ${ }^{5}$ Department of Neurology, Davos Hospital, Yongin, Republic of Korea
\end{abstract}

\subsection{6/oemed-2018-ICOHabstracts.102}

Introduction Night work is associated with shorter sleep and greater chronic disease risk compared with day work, and older shiftworkers report even greater difficulty sleeping compared with younger workers. We tested a sleep and circadian rhythm intervention to examine whether it improved sleep duration and cortisol, a marker of physiological stress.

Methods 26 healthy adults $(57.6 \pm 3.9$ y) who were not shiftworkers participated. Four laboratory Day shifts were followed by four Night shifts. Participants slept at home and maintained $\sim 8$ hour sleep schedules for a week before study and on Day shifts. After the first Night shift, participants were randomised into groups with different sleep instructions: control ad lib sleep $(n=9) ; 8$ hour evening sleep plus a light intervention $(n=9) ; 8$ hour evening sleep $(n=5)$. The evening sleep groups were instructed to get into bed between 1-2 pm and remain in bed attempting to sleep for 8 hour. Sleep was monitored by actigraphy. At the start of the 4th Day shift and end of the 4th Night shift, a blood sample was taken for cortisol assay.

Results The sleep duration and cortisol level of the groups were not different at baseline. The 8 hour evening sleep groups showed similar sleep durations following night and day shifts, while the control group had shorter sleep $(p<0.001)$. At the end of the 4th Night shift, the 8 hour evening sleep groups had significantly lower cortisol levels compared with the control group $(\mathrm{p}<0.02)$.

Discussion Our preliminary data indicate an 8 hour scheduled evening sleep episode after night shifts results in longer sleep in older shiftworkers, and this was associated with lower cortisol levels. While this remains to be tested in actual night workers, it suggests that the sleep intervention may have implications for improved health outcomes in older shiftworkers.

Grants R01AG044416， UL1TR001102， T32HL007901 from the NIH; Dancook University (to JHK).

\section{$1617 d$ SHIFTWORK AND METABOLIC HEALTH RISKS - WHAT DOES THE LITERATURE CONCLUDE?}

${ }^{1}$ Karin I Proper, ${ }^{2}$ Daniëlla van de Langenberg, ${ }^{1}$ Wendy Rodenburg, ${ }^{2}$ Roel CH Vermeulen, ${ }^{3}$ Allard J van der Beek, ${ }^{1}$ Harry van Steeg, ${ }^{1}$ Linda WM van Kerkhof. ${ }^{1}$ National Institute for Public Health and the Environment, Bilthoven, The Netherlands; ${ }^{2}$ Institute for Risk Assessment Sciences, Utrecht University, The Netherlands; ${ }^{3}$ Department of Public and Occupational health, VU University Medical Centre, Amsterdam, The Netherlands

\subsection{6/oemed-2018-ICOHabstracts. 103}

Introduction Although the metabolic health effects of shift work have been extensively studied, a systematic synthesis of the available research is lacking. This review aimed to systematically summarise the available evidence of longitudinal studies linking shift work with metabolic risk factors.

Methods A systematic literature search was performed. Studies were included if (1) they had a longitudinal design; (2) shift work was studied as the exposure; and (3) the outcome involved a metabolic risk factor, including anthropometric, blood glucose, blood lipid, or blood pressure measures. Eligible studies were assessed for their methodologic quality. A best evidence synthesis consisting of three levels of evidence was used to draw conclusions per outcome: strong, moderate or insufficient evidence.

Results Thirty-nine articles describing 22 studies were included. Strong evidence was found for a relation between shift work and increased body weight/BMI, risk for overweight, and impaired glucose tolerance. For the remaining outcomes (waist circumference, blood lipids, and blood pressure), there was insufficient evidence.

Discussion Shift work seems to be associated with body weight gain, risk for overweight, and impaired glucose tolerance. Overall, lack of high-methodologic quality studies and inconsistency in findings led to insufficient evidence in assessing the relation between shift work and other metabolic risk factors. To strengthen the evidence, more high-quality longitudinal studies that provide more information on the shift work 
schedule (e.g., frequency of night shifts, duration in years) are needed. Further, research to the (mediating) role of lifestyle behaviours in the health effects of shift work is recommended, as this may offer potential for preventive strategies.

\section{7e WORKLOAD AND EARLY FUNCTIONAL AGEING AMONG HOSPITAL WORKERS}

${ }^{1}$ Maria Carmen Martinez, ${ }^{2}$ Maria do Rosário Dias de Oliveira Latorre, ${ }^{3}$ Frida Marina Fischer. 'Independent consultant in Epidemiology in Occupational Health. WAF Informática, São Paulo, Brazil; ${ }^{2}$ Department of Epidemiology, School of Public Health, University of São Paulo, Brazil; ${ }^{3}$ Department of Environmental Health, School of Public Health, University of São Paulo, Brazil

\subsection{6/oemed-2018-ICOHabstracts. 104}

Introduction Early functional ageing - EFA is the progressive work ability - WA impairment preceding the chronological ageing. Hospital work is characterised by physical and mental workloads arising from work stressors that can influence functional ageing. The aim of this follow-up study was to assess the association between psychosocial work stressors and functional ageing in a group of Brazilian hospital employees.

Methods This is a 3 year follow-up (2009-2012) study of 423 hospital workers of São Paulo, Brazil. We included only workers with excellent/good WA at the baseline. At baseline workers filled a form on sociodemographic, lifestyle, and occupational questions including the Brazilian versions of Job Stress Scale, Effort-Reward Questionnaire, Work-Related Activities That May Contribute To Job-Related Pain and/or Injury, and Work Ability Index. Moderate/poor WA were considered as EFA. Changes from excellent/good to moderate/poor WA were the dependent variable. We used logistic regression models adjusted for potential confounders.

Results The average age was 36.0 y $(\mathrm{SD}=8.3), 30.7 \%$ were over $40 \mathrm{y}, 72.1 \%$ were females. At the end of follow-up the exposure to work stressors had worsened: job strain (20.3\%), social support (22.7\%), effort-reward imbalance (18.7\%), overcommitment (18.4\%) and Work-Related Activities That May Contribute To Job-Related Pain and/or Injury (13.9\%). Eighteen percent of the workers shifted to moderate or poor WA. High levels of exposure to psychosocial work stressors were significantly associated with decreased work ability: job strain $(\mathrm{OR}=2.81)$ and effort-reward imbalance $(\mathrm{OR}=3.21)$.

Discussion Work stressors were risk factors for work ability. The results showed the need for interventions to maintain hospital employees' work ability. Such strategies have implications for institutional and social policies to prevent early functional ageing.

\section{$1617 f$ COPING WITH SHIFTWORK: INDIVIDUAL AND TEAM STRATEGIES FOR HEALTH AND SAFETY}

1) Dorrian*, ${ }^{1} S$ Centofanti, ${ }^{2} \mathrm{~A}$ Colella, ${ }^{2} \mathrm{~L}$ Devine, ${ }^{2} \mathrm{C}$ Dingle, ${ }^{2} \mathrm{H}$ Galindo, ${ }^{2} \mathrm{~S}$ Pantelios, ${ }^{2} \mathrm{G}$ Brkic, ${ }^{3} \mathrm{C}$ Bull, ${ }^{3} \mathrm{~T}$ Almond, ${ }^{3} \mathrm{~V}$ Dhillon, ${ }^{1} \mathrm{MA}$ Carskadon, ${ }^{1} \mathrm{~S}$ Banks. ${ }^{1}$ University of South Australia, Adelaide, Australia; ${ }^{2}$ SA Health, Adelaide, Australia; ${ }^{3}$ CSIRO, Adelaide, Australia

\subsection{6/oemed-2018-ICOHabstracts. 105}

Introduction Shiftwork can negatively impact on performance, safety and health. Importantly, while some shiftworkers are profoundly affected, others cope very well. The aim of this project was to identify healthy shiftworkers (across measures of physical, behavioural and psychosocial health) in a group of Australian nurses and midwives, and to examine factors that may contribute to health across working life.

Methods This was a mixed methods study, combining survey, interview and biological measurements. Participating hospital nurses and midwives $(\mathrm{n}=96, \mathrm{f}=85$, age $=44.3 \pm 12.6 \mathrm{y}$, shiftwork experience $=18.9 \pm 12.7$ y) completed the Standard Shiftwork Index, which includes measures of health, social, domestic and work disruption, and coping styles. Participants could also participate in an interview $(n=22)$, and/or provide a saliva sample $(n=45)$, to measure telomere length (if our DNA were shoelaces, telomeres would be the aglets).

Results Cluster analysis revealed a 'Healthier' and a 'Less Healthy' cluster, with significantly worse scores across all health variables $(\mathrm{p}<0.05)$. Controlling for gender, age and work hours, the odds of being 'Less Healthy' were significantly $(p<0.05)$ : reduced for experienced shiftworkers $(\geq 20 y$ versus $<20 \mathrm{y}, \mathrm{OR}=0.16$ ); reduced by engaged coping style $(\mathrm{OR}=0.92)$; and increased by disengaged coping style $(\mathrm{OR}=1.20)$. Strategies for coping were aimed at promoting wakefulness/sleep at biologically difficult times, sustaining safe performance at work and while driving, and maintaining healthy diet, exercise and relationships. Strategies included the use of stimulants and sedatives, changes in composition/timing of food intake, changes in timing/distribution of sleep, advances/delays in undertaking safety-critical activities, and contributing to a culture of social support. Correlations between age and telomere length were negative in less experienced $(<20 \mathrm{y}$, $r=-0.22)$ and positive in more experienced $(\geq 20 \mathrm{y}, r=0.85)$ shiftworkers.

Conclusion Findings provided evidence of healthy ageing in experienced shiftworkers that was linked to coping style. Studying those who cope well provides an evidence-base for teaching successful behavioural strategies to improve worker health and safety.

\section{$1617 \mathrm{~g}$ ASSOCIATION OF INSOMNIA AND FATIGUE DUE TO SHIFT WORK IN MIDLIFE AND MOBILITY LIMITATIONS OVER 28 YEARS OF FOLLOW-UP}

${ }^{1,2} \mathrm{KC}$ Prakash*, ${ }^{1,2}$ Subas Neupane, ${ }^{3}$ Päivi Leino-Arjas, ${ }^{3}$ Mikko Härmä, ${ }^{1,2}$ ClasHåkan Nygård. ${ }^{1}$ Faculty of Social Sciences, Health Sciences, University of Tampere, Tampere, Finland; ${ }^{2}$ Gerontology Research Centre, University of Tampere, Tampere, Finland; ${ }^{3}$ Finnish Institute of Occupational Health, Helsinki, Finland

\subsection{6/oemed-2018-ICOHabstracts. 106}

Objectives We aimed to investigate the long-term association of insomnia and fatigue due to shift work (with and without night shifts) in midlife and mobility limitations (ML) among initially middle-aged subjects followed over 28 years.

Methods The Finnish Longitudinal Study on Ageing Municipal Employees (FLAME) was conducted by the Finnish Institute of Occupational Health from 1981 to 2009 among 6257 municipal workers. This study is based on the latest available response on ML questionnaires either from 1992 or 1997 or in $2009(n=4704)$. International Classification of Functioning (ICF) was used to code the nine mobility tasks included in the ML. Insomnia and fatigue due to shift work were assessed using the yes/no questions in the baseline. The Incidence Rate Ratio (IRR) and 95\% Confidence Interval (CI) for the association of insomnia and fatigue due to shift work and ML were assessed by using mixed Poisson regression. The results are presented separately for women and men in shift work with and without night shifts. 